\title{
Quand l'élève gère son temps: le rapport au temps dans le travail autonome à l'école primaire
}

\section{Héloïse Durler}

Cette contribution aborde les contradictions temporelles qui s'imposent aux élèves et aux enseignants de l'école primaire dans les dispositifs de travail autonome. Elle examine les formes de rapport au temps sous-tendues par ces modalités de travail. Basée sur une enquête ethnographique menée dans un établissement scolaire primaire genevois, elle met en évidence que les élèves ont à établir un rapport gestionnaire au temps, source à la fois d'émancipation et de contrôle. Elle considère comment les catégories temporelles sont utilisées pour naturaliser les difficultés comportementales et cognitives des élèves, alors même qu'on peut les interpréter comme le produit de socialisations temporelles familiales inégalement légitimes et rentables à l'école.

Quelles formes de rapport au temps les élèves de l'école primaire ont-ils à mettre en œuvre lorsqu'ils travaillent? Michel Verret notait en 1975 déjà que le temps scolaire «requiert des agents scolarisés, outre la disponibilité temporelle, les aptitudes à la régularité et à la ponctualité, des capacités de prévision et de planification» (pp. 212-213). Les résultats obtenus à travers une recherche ethnographique (Durler, 2015) nous permettent d'avancer que la valorisation de certaines formes de travail - en particulier lorsque les élèves doivent réaliser de manière autonome leurs activités - contribue à complexifier les exigences scolaires concernant l'usage du temps.

Après avoir exposé dans une première partie le cadre théorique et la méthodologie de la recherche sur lesquels se basent les développements proposés ici, on examinera dans une deuxième partie les attentes contenues dans les dispositifs de travail autonome concernant l'utilisation du temps. On verra que ces modalités de travail introduisent plus de flexibilité temporelle et accordent davantage de marges de manœuvre aux élèves, tout en augmentant, pour les enseignantes et les enseignants, les possibilités de contrôler et d'évaluer l'activité des élèves. On examinera en particulier comment les supports écrits qui accompagnent les moments de travail en autonomie amènent tout à la fois à un usage spécifique du temps et à une responsabilisation accrue des élèves dans l'organisation de leurs activités. Dans une troisième partie, on considérera comment ces formes 
de rapport au temps, présupposées par les dispositifs pédagogiques, participent à l'élaboration, chez les enseignantes et les enseignants, de catégories temporelles d'évaluation de l'activité et des performances des élèves, traduisant les contradictions inhérentes aux dispositifs de travail autonome.

\section{Une ethnographie des dispositifs pédagogiques}

La présente contribution se base sur les résultats d'une enquête ethnographique menée durant près de trois ans dans un établissement scolaire primaire genevois. Son objectif était d'identifier les types de difficultés scolaires auxquelles sont confrontés les élèves lors des premières années de scolarité. Il s'agissait, au-delà des constats statistiques montrant que les inégalités de réussite scolaire en fonction de l'origine sociale des élèves sont en place dès l'entrée à l'école (Cayouette-Remblière, 2016; Duru-Bellat, 2002; Felouzis \& Goastellec, 2014), de comprendre les processus par lesquels ces difficultés se construisent, au quotidien. À la croisée d'une sociologie dispositionnaliste (Bourdieu 1994; Lahire, 1998) et interactionniste (Becker, 1985; 2002, Hughes, 1996), notre approche place la focale sur l'analyse des pratiques individuelles, des interactions et apprentissages (attendus ou non) propres au contexte de la classe, tout en considérant les individus comme porteurs de dispositions, de manières d'être, de parler, de penser, etc., construites à l'intérieur de différentes sphères de socialisation (scolaire, familiale, amicale, sportive, artistique, etc.) (Darmon, 2006). On s'est ainsi intéressé aux processus de socialisation scolaire, en cherchant à identifier les formes d'organisation du travail en classe, les pratiques d'enseignement, les supports pédagogiques privilégiés et leurs modalités d'appropriation par les élèves.

La volonté de se concentrer sur les pratiques quotidiennes a impliqué une immersion prolongée dans un terrain de taille réduite, la réalisation d'observations systématiques, répétitives, et d'entretiens approfondis (Beaud \& Weber, 2010). C'est pourquoi la démarche d'enquête par observation participante a été choisie, afin d'approcher le travail quotidien des élèves et des enseignants. L'enquête s'est déroulée entre 2006 et 2008 et a compris des observations de classes et des entretiens avec le personnel enseignant, des élèves et leurs parents. Elle a été réalisée dans un établissement accueillant au total un peu plus de 300 élèves distribués dans quinze classes de degrés primaires. Situé dans un quartier urbain, l'établissement comprenait une surreprésentation d'élèves issus des milieux populaires et d'origine étrangère, par rapport aux taux moyens des écoles de la ville de Genève. Plus de 200 heures d'observations ont été réalisées dans neuf classes allant de la $2^{\mathrm{e}}$ primaire $(2 \mathrm{P})$ à la $5^{\mathrm{e}}$ primaire $(5 \mathrm{P})$ auprès d'élèves âgés de 5 à 10 ans. Des entretiens semi-directifs de longue durée (deux fois deux heures par interviewé) ont été menés avec cinq enseignants, de même que des observations de réunions d'enseignants, formelles (conseils des maîtres, réunions "pédago- 
giques», réunions de "décloisonnement», entretiens entre enseignants et parents, etc.) ou informelles (repas de midi, pauses café, surveillances de récréation, etc.). L'enquête a également compris le suivi intensif de quatre enfants dans leur classe (observations) et dans leur famille (observations), par le truchement d'une activité d'aide aux devoirs à domicile, tout au long de l'enquête. Une série d'entretiens semi-directifs auprès de dix élèves de $4^{\mathrm{e}}$ primaire $(4 \mathrm{P})$ et auprès de neuf familles de milieu populaire, ainsi que des discussions informelles avec les enseignants réguliers, avec les enseignants de rythmique ou de gymnastique, avec des intervenants extérieurs (par exemple, artistes intervenant dans la classe) ou des professionnels non enseignants (par exemple, logopédistes) au sujet des enfants suivis ont permis de compléter le corpus de données.

Sur la base des retranscriptions des notes d'observation, consignées dans un journal de terrain ${ }^{1}$, et des entretiens, une analyse de contenu a été réalisée dans le but, d'une part, de dégager une typologie des dispositifs pédagogiques (Bonnéry, 2009; 2011) privilégiés dans les classes et d'autre part d'identifier, à travers leur description, les pratiques et stratégies des acteurs à l'intérieur de ces dispositifs. La notion de dispositif (Foucault, 1975; Lahire, 2005) renvoie ici au réseau ou aux relations que l'on peut identifier d'un ensemble d'éléments hétérogènes qui produit des effets sur les actions individuelles. Elle permet de considérer simultanément l'organisation du travail, l'utilisation du temps et de l'espace, l'usage des outils pédagogiques, les conceptions de l'apprentissage, etc., et d'identifier comment cet ensemble d'éléments oriente les comportements individuels (les rendent possibles, les interdisent, définissent des usages plus ou moins légitimes des supports pédagogiques, etc.).

Les données recueillies sur le terrain apparaissent ainsi sous deux statuts distincts. Premièrement, à travers la présentation des dispositifs, les extraits du corpus sélectionnés (observations et entretiens) constituent une "condensation" des données les plus récurrentes et ont été choisis en fonction de leur «force d'illustration" d'un ensemble de données convergentes. Deuxièmement, dans l'analyse des pratiques et stratégies individuelles, l'attention s'est portée sur ce que font les acteurs avec ces dispositifs, dans une perspective interactionniste. L'analyse du matériel a alors été conduite en fonction des écarts, contradictions et tensions qui, même s'ils peuvent apparaître comme minoritaires ou singuliers, sont alors considérés comme autant de symptômes ou d'indicateurs permettant de comprendre la dynamique interne des dispositifs. L'objectif était d'envisager, à partir de la description et de l'analyse des pratiques, comment se traduisaient, au niveau micro, au sein des dispositifs, des écarts, contradictions et tensions entre définitions sociales de l'enfance (Chamboredon \& Prévot, 1973) et pratiques éducatives inégalement présentes dans les différents milieux sociaux.

L'analyse du matériau d'enquête a permis de mettre en évidence que l'autonomie des élèves apparait à la fois comme une visée explicite et pratique de la socialisation scolaire et comme une catégorie de justification se traduisant dans des supports pédagogiques (fiches individuelles, «plan de travail», ateliers autocor- 
rectifs, etc.) et influençant les interactions entre enseignants et élèves (valorisation de postures de tâtonnement et de recherche chez les élèves, mise en retrait de l'enseignant, demandes d'auto-évaluation formulées vis-à-vis des élèves, etc.). Elle a permis d'identifier les attentes récurrentes contenues par les dispositifs à l'égard des élèves. Ceux-ci sont par exemple invités à se positionner en tant que «sujets réflexifs» de leurs apprentissages et de leur expérience, à prendre l'habitude de se questionner et de transformer leur «vécu» en occasion d'apprentissage. De la même manière, ils ont à se remettre en question, notamment à travers des pratiques d'autocorrection ou d'auto-évaluation, engageant une conception de l'erreur comme levier d'apprentissage. Les élèves ont de surcroît à prendre l'habitude de planifier leur travail, à utiliser des supports (tableaux, calendriers, «maisons des apprentissages», etc.) participant à inscrire l'avancement du travail dans le temps et à objectiver les apprentissages réalisés. L'analyse a également mis en évidence des procédés de responsabilisation à l'œuvre dans les dispositifs pédagogiques: en augmentant les possibilités de choix pour les élèves et en leur déléguant une partie du contrôle de leurs activités, les dispositifs leur demandent d'endosser une part accrue de la responsabilité de l'avancement du travail scolaire. C'est enfin la "participation» des élèves et leur «engagement» dans les activités qui sont attendus: les élèves doivent manifester leur motivation, voire leur plaisir à entrer dans les activités scolaires.

L'enquête a permis d'identifier les principales contradictions de ces dispositifs, notamment lorsqu'ils s'adressent à des enfants issus de milieux populaires. Les attentes implicites qu'ils contiennent les mettent souvent en difficulté, tandis que les ressources nécessaires à la réalisation du travail sont le plus souvent considérées comme des «allant de soi» et ne font pas l'objet d'un enseignement explicite. La contradiction principale réside dans le fait que ce sont envers les élèves objectivement les plus éloignées de l'univers scolaire, qui possèdent le moins de ressources (en temps, en capital culturel et économique) pour se «mobiliser» dans le sens attendu, que les injonctions (à la motivation, à l'engagement, à l'autonomie, etc.) se font les plus pressantes. On développera ici les principales tensions relatives à l'usage du temps dans les dispositifs pédagogiques étudiés.

\section{Le temps du travail en autonomie: entre liberté et contrôle}

\section{Un rapport gestionnaire au temps}

Dans les classes observées, les élèves sont régulièrement mis en situation de devoir organiser individuellement leur travail, gérer un ensemble de tâches, planifier une série d'exercices, etc., et ce dès les premières années de scolarité. Les élèves ont alors à organiser leur travail à l'intérieur d'une période délimitée, apprendre à passer d'une activité à une autre sans intervention de l'enseignant, 
ne pas «perdre de temps», au risque de se mettre en retard, respecter les délais impartis (une, deux, voire trois semaines), etc.

"Les contrats de travail, on appelle ça comme ça. Dans une fourre, on leur met plusieurs fiches, on leur donne quarante-cinq minutes par jour et au bout de huit jours d'école, ils doivent avoir tout terminé. C'est un petit contrat, comme ça» (Entretien avec Françoise, enseignante)

Ces dispositifs posent un cadre temporel à l'intérieur duquel une autonomie est laissée aux élèves: un espace de choix leur est proposé, une liberté dans l'ordre des tâches à réaliser, dans l'organisation personnelle du travail, dans la méthode de travail ou encore les supports utilisés.

«Sylvie - Alors on peut passer au plan de travail. Ils ont des périodes dans la semaine, pour le faire. Ça leur permet de gérer un peu leur travail. Ils ne sont pas obligés de faire, tous, les mêmes fiches en même temps. Ils savent que, maintenant, on a fait un planning, on fait le plan de travail, et qu'ils sont libres de choisir la feuille qu'ils veulent, dans le plan» (Extrait du journal de terrain, classe de Sylvie et Nelly, 3P-4P, réunion de parents)

Les dispositifs de travail autonome sont souvent présentés par les enseignants comme le moyen d'adapter le travail aux «rythmes» des enfants. C'est ainsi un rapport "expressif» (Plaisance, 1986) et individualisé au temps qui est mis en avant, dans lequel le fait de laisser la possibilité à l'élève d'user «librement» de son temps pour réaliser son travail apparaît comme un atout.

«Daphné- On essaie de respecter le rythme de l'enfant. On différencie, après, au niveau des fiches, parce que chaque enfant est différent» (Extrait du journal de terrain, classe de Fabio et Daphné, 2P-3P, réunion de parents)

On retrouve dans les dispositifs de travail autonome les formes de rapport au temps plus généralement attendues à l'école, comme le rapport ascétique au temps (Henri-Panabière, 2008, novembre), dans lequel les élèves doivent savoir reporter un plaisir immédiat et persévérer dans un effort en vue d'une gratification ultérieure, ou encore le rapport structuré au temps, dans lequel le «moment» (Darmon, 2001) comme unité de temps, de lieu et d'activité, joue un rôle central. Le temps scolaire est un temps divisé, découpé et les élèves doivent comprendre "qu'il y a un temps pour chaque chose» et faire les choses au «bon moment». Les "emplois du temps» traduisent cette structuration: affichés sur le tableau noir, sur un mur de la classe, ou encore annoncés en début de journée par l'enseignant, ils ont pour fonction de donner aux élèves les moyens d'anticiper les différentes activités qui vont survenir au cours de la journée.

Plus spécifiquement, les modalités de travail autonome demandent aux élèves d'établir un rapport gestionnaire et productif au temps: savoir tenir des délais, respecter un emploi du temps, définir des priorités dans les tâches à réaliser, etc. Ils doivent plus généralement occuper «utilement» leur temps, le rentabiliser, le «mettre à profit» pour réaliser des activités qui ont une légitimité scolaire 
(les activités «d'occupation» du temps «libre» doivent comporter une fonction éducative, comme la lecture d'un livre, jeux pédagogiques, dessins, etc.) ou pour «avancer» dans leur travail:

"Véronique - Qui a déjà fait un plan de travail? Qui peut m’expliquer? Ça consiste en quoi, un plan de travail? [...] Une élève - Ça sert à quand on a un petit peu de temps libre, on fait du plan de travail» (Extrait du journal de terrain, classe de Véronique et Cécile, 3P-4P)

Les élèves doivent donc prendre une série d'initiatives et de décisions et, partant, prendre sous leur responsabilité une partie du contrôle de leurs activités. Les dispositifs de travail autonome encouragent ainsi les élèves à conduire leurs activités sur le mode du projet, la capacité à construire et conduire des projets devenant centrale ${ }^{2}$. En laissant des choix aux élèves, en leur accordant une marge de manœuvre dans la gestion de leur travail, en leur demandant de négocier les règles de l'activité en classe, on dispose de puissants moyens d'engagement des élèves dans les tâches scolaires. L'élève qui a «librement» choisi une activité est fortement contraint à la réaliser jusqu'au bout. Un "piège pédagogique» (Lahire, 2005, p. 330) se referme sur lui: il ne peut pas ne pas réaliser complètement ce qu'il a "choisi» de faire, il est considéré comme «responsable» d'un choix qu'il doit «assumer» 3 .

Ainsi, le rapport gestionnaire au temps qu'il est demandé aux élèves d'établir dans leur travail va de pair avec une responsabilisation croissante: en leur laissant la possibilité de gérer librement, sur des périodes temporelles délimitées, une partie de leurs activités, les élèves ont à prendre à leur charge un ensemble de décisions pouvant influencer leur travail et leur réussite scolaire.

\section{La traçabilité du temps}

Si l'on ne peut comprendre le rapport au temps scolaire sans prendre en compte les formes de travail valorisées dans les classes, l'utilisation des supports écrits qu'elles entraînent semble jouer un rôle de premier plan. En effet, le double mouvement d'émancipation et de contrôle qui traverse les dispositifs de travail autonome est à mettre en lien avec l'ampleur prise par les supports écrits dans le travail scolaire, dès les premières années de scolarité. La montée en responsabilité des élèves dans leur travail s'accompagne de procédures leur demandant de noter, recenser, décrire leurs pratiques et, partant, d'accorder autant, voire davantage d'importance à la visibilité du travail qu'à l'activité d'apprentissage en elle-même. De la même manière, le temps «mis à disposition» de l'élève doit être «rapporté», justifié, et donner lieu à une activité "traçable» et évaluable. Les élèves ont à mettre en œuvre une série d'opérations complexes (anticiper, planifier, prioriser, mais aussi se repérer dans un calendrier, un horaire, se remémorer des activités passées pour les évaluer, etc.). Par exemple, lors de l'utilisation du "plan de travail», les élèves ont, à partir d'un passage en revue de l'ensemble des fiches à réaliser, la possibilité de poser des questions à l'enseignante ou à l'enseignant, 
c'est-à-dire anticiper les problèmes et se projeter dans le travail à réaliser. De la même manière, ce type de support permet aux enseignants de se rendre compte rapidement de l'état d'avancement du travail en consultant un tableau récapitulatif, sans avoir à questionner directement les élèves ou même à consulter les fiches réalisées. Les élèves doivent en effet mentionner dans un tableau le nombre de fiches réalisées, ainsi que la difficulté perçue des tâches demandées. On peut ici parler d'une "discipline de reporting", identifiée en sociologie du travail par Sylvie Monchatre (2004) à propos des transformations touchant le travail des ouvriers, convertis en "opérateurs» avec la mise en place de «démarches compétences» impliquant «une nouvelle manière d'énoncer les qualités du travail, en termes de compétence, d'autonomie, d'initiative» (p. 71) et des nouvelles pratiques de consignation par écrit des activités.

Les élèves ont également à apprendre à gérer des délais. Dans le contexte de l'utilisation du plan de travail, il est annoncé aux élèves que l'entier du travail demandé doit être réalisé sur une période définie, tandis que différentes pratiques soulignent cette délimitation dans le temps. Les enseignants peuvent par exemple indiquer par des cases sur le tableau noir le nombre total de moments dévolus au "plan de travail» et les cocher au fur et à mesure de la progression dans la semaine, mais il peut aussi être demandé aux élèves de noter sur un tableau personnel l'avancée chronologique de leur "plan de travail»:

"Quand ils ont fini un travail, ils mettent dans la boîte à corriger, et moi je corrige. Si c'est bon, ils ont le droit de colorier le petit soleil. Si c'est pas bon, on corrige. Et chaque fois qu'on a fini une période, ils mettent une croix. Comme ça, eux aussi savent où ils en sont, au niveau du temps.» (Entretien avec Maude, enseignante)

Ces opérations sont d'autant plus attendues qu'elles sont rendues nécessaires par la «révolution pédagogique silencieuse que représente la multiplication des supports écrits à l'école» (Joigneaux, 2014, p. 73). Davantage responsabilisés, les élèves se voient ainsi dotés de marges de manœuvre plus importantes et des choix leur sont laissés dans l'organisation de leur travail. Dans le même temps, l'utilisation accrue et précoce des supports écrits dans les dispositifs de travail autonome augmente chez les enseignants les possibilités de contrôle du travail et des comportements des élèves.

Comme l'ont montré les travaux de Cochoy, Garel et de Terssac (1998) sur l'introduction des normes ISO dans l'entreprise, l'essor de la forme écrite et le développement d'un "processus d'écriture généralisé» (p.674) au sein des organisations contiennent des possibilités émancipatoires. Un espace est en effet laissé à l'individu qui peut obtenir une reconnaissance écrite de son domaine de responsabilité. Dans le même temps, un contrôle plus serré peut s'exercer sur la consignation écrite des pratiques de travail. Ce double mouvement d'émancipation et de contrôle est à l'œuvre dans les classes observées: la multiplication des supports écrits donne à l'élève la possibilité de se faire une vision d'ensemble 
des tâches à réaliser et lui procure la maîtrise de l'ordonnancement de ces tâches, tandis que la mise à distance temporelle (entre la réalisation du travail et son contrôle par l'enseignant) demande un cadrage important de la part de l'enseignant, tant dans la préparation que dans la restitution du travail aux élèves. Les dispositifs pédagogiques de travail autonome permettent aux enseignants à la fois de "guider à distance» et de "suivre à la trace» (Joigneaux, 2013) les élèves dans leur travail, mais impliquent une préparation importante des activités en amont, comme me l'expliquent les enseignantes et les enseignants: «tout» doit être organisé, pour rendre les élèves autonomes (Extrait du journal de terrain, classe de Maude, 2P-3P), pour éviter le «flottement» des élèves dans les activités (Extrait du journal de terrain, classe de Sylvie et Nelly, 3P-4P). Les modalités d'utilisation de l'écrit, lorsqu'elles sont utilisées dans la conduite des pratiques, sont ainsi indissociables des formes relationnelles s'établissant entre les acteurs (Goody, 1979).

Ces dispositifs participent donc à former des individus «autonomes» aptes à s'insérer dans les dynamiques valorisées par le marché du travail actuel: responsabilisation accrue des individus, adaptabilité aux changements, flexibilité face aux conditions de travail fluctuantes, capacité d'auto-contrôle, etc. Ils introduisent les élèves aux «nouveaux cadres spatio-temporels de la vie quotidienne» (Boulin, Lallement, \& Volkoff, 2006). Avec les nouvelles formes d'organisation du travail émergent en effet de nouvelles temporalités, plus «individualisées», plus «libres» et mieux «choisies». La capacité à maîtriser son temps et à construire des projets se trouve socialement et scolairement valorisée. Dans le même temps, les élèves doivent porter une part des tensions inhérentes à ces nouvelles formes d'organisation du travail et faire face, pour certains, à des situations contradictoires. Au nombre de ces difficultés, il faut mentionner les attentes temporelles et cognitives contenues dans les modalités de travail scolaire autonome, posant problème aux élèves qui n'ont pas les ressources pour y répondre adéquatement.

\section{Le temps comme langage des hiérarchies scolaires}

Les catégories temporelles utilisées par les enseignants à travers les verdicts scolaires traduisent les contradictions des attentes curriculaires. En effet, alors que les dispositifs de travail autonome sont explicitement mis en place pour permettre aux élèves de réaliser «à leur rythme» les activités scolaires, les catégories utilisées par les enseignants dans leurs jugements des performances des élèves indiquent le contraire. La vitesse à laquelle les élèves effectuent le travail demandé reste un critère discriminant. Si les enseignantes et les enseignants souhaitent que les élèves réalisent le travail «à leur rythme», celui-ci doit tout de même suivre un certain «tempo scolaire»: 
«Le début d'année a été plutôt difficile, mais là, on a l'impression qu'il reprend son petit rythme de croisière où ça va. En tout cas, moi, [...] je vois qu'il est plus à son affaire. Enfin, que le fait d'être devant, il se sent plus concerné. On a l'impression qu'il reprend son rythme.» (Entretien avec Elisabeth, enseignante)

Ces observations rejoignent les constats faits par Keddie (1971/2007) dans l'enseignement secondaire, lorsqu'elle montre que les enseignants disent laisser à chaque élève la possibilité de "travailler à sa propre allure», mais que l'allure sur laquelle les élèves doivent se caler au final est bien celle des «bons élèves».

Les élèves eux-mêmes se comparent entre eux et distinguent clairement les élèves qui parviennent à réaliser «dans les temps» les activités demandées et ceux qui n'y parviennent pas.

"Une élève - Parfois, il y a des gens qui regardent dans les casiers et qui disent: "Eh, t’as pas terminé tes fiches." Parfois ils disent: “Oh, tout ce qu'il a à terminer!"» (Extrait du journal de terrain, classe d'Elisabeth, 3P-4P)

Il arrive fréquemment que les difficultés des élèves soient analysées comme un problème de rythme, considéré comme "extrêmement lent»:

«Bon, lecture, elle ne lit pas très bien non plus, elle ne comprend pas franchement ce qu'elle lit. Et la lenteur, elle est extrêmement lente, elle a un rythme extrêmement lent. Ça, je ne sais pas. Bon, on a tous des rythmes différents, mais c'est vrai que ça, c'est assez lourd, chez elle.» (Entretien avec Anne-Claire, enseignante d'appui)

La «lenteur» devient ainsi une catégorie naturalisée, un "problème» qui toucherait une partie des enfants. Certains enseignants se plaignent alors d'une population d'élèves particulière, selon eux en augmentation:

«On a quand même de plus en plus d'enfants qui ont besoin de plus de temps pour apprendre.» (Entretien avec Françoise, enseignante).

On distingue, en filigrane de ces propos, une référence à un élève «idéal», qui apprend et fait son travail selon une cadence «normale». C'est d'ailleurs par comparaison (à ce qui est attendu, aux comportements des autres élèves de la classe, etc.) qu'émerge la «déviance temporelle» de certains élèves:

«Fabio - Une autre élève qui me cause souci, au niveau scolaire, c'est [Nina], 3P. J'ai quand même passé une demi-heure à lui expliquer le $0+1$, alors qu'avec les autres 3P de ma classe, ça roulait au bout de 5 minutes.» (Extrait du journal de terrain, conseil des maitres)

Les faibles capacités ou temps de concentration des élèves sont également régulièrement invoqués par les enseignants:

«Fabio - Lui, il m'inquiète plus. Il reste dans son coin, il ne dit rien et il a un temps de concentration vraiment très réduit. Je n'ai vraiment pas l'impression qu'il comprend les consignes qu'on lui donne.» (Extrait du journal de terrain, conseil des maitres) 
Les solutions qui se présentent logiquement aux enseignants sont d'appeler les élèves à se "concentrer» (comme s'il s'agissait là d'un simple effet de la volonté individuelle) et de laisser "plus de temps» aux élèves "qui en ont besoin». Ce temps supplémentaire peut toutefois apparaître comme une "offre» à double tranchant pour les élèves qui, non seulement ne parviennent pas davantage à réaliser ce qui leur est demandé de faire, mais se trouvent de surcroît accusés de ne pas avoir su saisir la "chance» ainsi offerte.

«Quand l'enseignante vient vers Ricardo pour voir ce qu'il fait (elle lui a laissé plus de temps que les autres pour terminer l'épreuve cantonale de mathématiques), il est en train d'écrire son prénom et de dessiner sur un coin de la première page. "Non, là, maintenant, Ricardo, j'en ai marre. Si je te laisse plus de temps, c'est pas pour gribouiller. Ricardo, les résultats parlent, pas besoin d'en rajouter"» (Extrait du journal de terrain, classe d'Elisabeth, 3P-4P)

Le «temps supplémentaire» peut tout simplement être renvoyé hors de la classe, les élèves «lents» ont alors à terminer chez eux le travail qui n'a pas été fini en classe. Ces enfants sont ainsi soumis à un régime de double peine: non seulement ils n'arrivent pas à effectuer le travail qui leur est demandé, mais en plus ils ont à le faire en dehors du contexte de la classe, seuls, à la maison. Or, on peut supposer qu'ils n'auront guère plus de facilité à réaliser hors de la classe ce qu'ils n'arrivaient pas à travailler dans la classe.

Ainsi, lorsque les élèves rencontrent des difficultés dans leur travail, le discours sur le «respect des rythmes» induit un glissement vers une perception des élèves qui seraient, par nature, plus ou moins «lents» ou capables de rester concentrés sur une période déterminée. Les difficultés d'apprentissage sont alors moins interprétées comme des difficultés cognitives, liées à l'acquisition des savoirs et des compétences scolaires, et davantage comme des «difficultés temporelles», renvoyant à la nature de l'élève (à sa "personnalité", à son "caractère»), sur laquelle les possibilités d'action de l'enseignant sont limitées.

Cette interprétation tend à faire oublier que tous les élèves ne sont pas égaux face aux attentes scolaires en matière de gestion du temps. Des travaux ont à cet égard montré comment les conditions matérielles d'existence et les pratiques familiales d'écriture influaient sur la constitution d'un rapport au temps plus ou moins favorable à la réussite scolaire, dans la mesure où elles autorisent des représentations variables de l'avenir (voir Beaud, 1997; Lahire, 1995; Millet \& Thin, 2005; Terrail, 1984). Lorsque l'organisation du travail en classe comprend la prise en charge par les élèves eux-mêmes d'une partie des activités, on peut supposer que certaines manières d'appréhender le temps, construites dans la sphère familiale, deviennent d'autant plus «rentables» ou "payantes» dans le cadre scolaire. 


\section{Conclusion}

Avec l'essor des dispositifs de travail autonome dans les classes primaires, c'est un rapport gestionnaire au temps qui est attendu, dans la mesure où les élèves ont à réaliser des opérations de planification, d'organisation, mais aussi d'évaluation de leurs activités scolaires. Cette temporalité scolaire renvoie au type de rapport au temps socialement valorisé dans les nouvelles formes d'organisation du travail, plus «flexibles», plus «individualisées», et correspond aux cadres spatio-temporels de la vie quotidienne des catégories sociales moyennes et supérieures. L'usage valorisé du temps dans les dispositifs de travail autonome n'est pas sans évoquer les pratiques de management des dernières décennies, le travail par projet, les techniques de responsabilisation des travailleurs, etc.

Se pose alors la question de l'éloignement de ces temporalités des autres catégories sociales, dont les conditions d'existence précaires, la non-insertion sur le marché de l'emploi ou l'occupation de postes aux horaires désynchronisés par rapport aux rythmes sociaux dominants (travail de nuit, horaires fragmentés, irréguliers, etc.) ne permettent pas d'établir un rapport au temps socialement valorisé. Les enfants issus des milieux précarisés ont de moindres possibilités de construire des dispositions temporelles «rentables» dans le contexte de l'école et de ses dispositifs de travail, portant à conséquence sur le plan des apprentissages scolaires. De plus, l'origine des difficultés des élèves tend à être naturalisée au moyen de catégories temporelles (le rythme et le temps de concentration) faisant porter aux individus (à leur caractère, à leur personnalité, etc.) la responsabilité de leurs échecs. En les percevant comme faiblement capables de concentration ou «lents» (à se mettre au travail, à le réaliser, etc.), on oublie que ces comportements sont les produits de socialisations, construits au sein de contextes familiaux parfois très éloignés de l'école et de ses réquisits. De la sorte, on peut faire l'hypothèse que l'école contribue à l'apparition de nouvelles doxas temporelles (injonction à se projeter, à maîtriser le temps, etc.) qui, s'imposant à tous, participent à délégitimer les temporalités des fractions sociales les plus démunies.

\section{Notes}

1 Support central de la collecte de données, le journal de terrain permet de consigner et de développer, à la fin de chaque séance d'observation, les notes prises «sur le vif» sur le terrain par la chercheuse. La tenue du journal de terrain constitue ainsi la trace du travail d'enquête, et lui permet d'amorcer, au moment de la reprise et de la réécriture des notes, l'analyse des phénomènes observés. Il est également le lieu d'une «auto-analyse» permettant aux chercheurs de réfléchir à leur rapport au milieu observé.

2 À cet égard, plusieurs travaux démontrent comment la notion de projet devient à la fois un outil éducatif et un instrument de classement des individus, notamment dans les dispositifs d'insertion (Faure \& Garcia, 2005; Mauger, 2001)

3 Ce qui n'est pas sans rappeler les constats des travaux réalisés en psychologie sociale sur l'engagement (Joule \& Beauvois, 2009, 2014). Leurs auteurs ont bien mis en évidence que la mise en place d'un "contexte de liberté», laissant apparemment la liberté à l'individu de choisir ou non d'adopter un comportement, comporte de forts effets d'engagement. 


\section{Bibliographie}

Beaud, S. (1997). Un temps élastique. Étudiants des cités et examens universitaires. Terrain, 29, 43-58.

Beaud S. \& Weber F. (2010). Guide de l'enquête de terrain (nouvelle éd. augmentée). Paris: La découverte

Becker H.S (1985). Outsiders. Etudes de sociologie de la déviance. Paris: Métailié.

Becker H. S. (2002). Les ficelles du métier. Comment conduire sa recherche en sciences sociales. Paris: La découverte.

Bonnéry, S. (2009). Scénarisation des dispositifs pédagogiques et inégalités d'apprentissage. Revue française de pédagogie, 167, avril-mai-juin, 13-23.

Bonnéry, S. (2011). Sociologie des dispositifs pédagogiques: structuration matérielle et technique, conceptions sociales de l'élève et apprentissages inégaux. In J.-Y. Rochex \& J. Crinon (Éd.), La construction des inégalités scolaires. Au cour des pratiques et des dispositifs d'enseignement (pp. 133-146). Rennes: Presses Universitaires de Rennes.

Boulin, J.-Y., Lallement, M. \& Volkoff, S. (2006). Introduction - Flexibilité, disponibilité et nouveaux cadres spatio-temporels de la vie quotidienne. Temporalités, 4, 1-5.

Bourdieu, P. (1977) Algérie 60. Structures économiques et structures temporelles. Paris: Editions de Minuit.

Bourdieu P. (1994) Raisons pratiques. Sur la théorie de l'action. Paris: Le Seuil.

Cayouette-Remblière, J. (2016). L'école qui classe. 530 élèves du primaire au bac. Paris: PUF.

Chamboredon, J.-C. \& Prévot, J. (1973). Le «métier d'enfant»: définition sociale de la prime enfance et fonctions différentielles de l'école maternelle. Revue française de sociologie, 14, 295-335.

Cochoy, F., Garel, J.-P. \& De Terssac, G. (1998). Comment l'écrit travaille l'organisation: le cas des normes ISO 9000. Revue française de sociologie, 39, 673-699.

Darmon, M. (2001). La socialisation, entre famille et école: observation d'une classe de première année de maternelle. Sociétés \& Représentations, 11, 517-538.

Darmon, M. (2006). La socialisation. Paris: Armand Colin.

Durler, H. (2015). L'autonomie obligatoire. Sociologie du gouvernement de soi à l'école. Rennes: Presses Universitaires de Rennes.

Duru-Bellat, M. (2002). Les inégalités sociales à l'école: Genèse et mythes. Paris: PUF.

Faure, S. \& Garcia, M.-C. (2005). Culture hip-hop. Jeunes des cités et politiques publiques. Paris: La dispute.

Felouzis, G. \& Goastellec, G. (Éd.) (2015). Les inégalités scolaires en Suisse: école, société et politiques éducatives. Berne: Peter Lang.

Foucault, M. (1975). Surveiller et punir. Naissance de la prison. Paris: Gallimard.

Joigneaux, C. (2013). Littératie, forme et inégalités scolaires: le cas de la «scolarisation» de l'école maternelle. Le français aujourd'hui, 183, 41-50.

Joigneaux, C. (2014). L'autonomie à la maternelle: un nouvel idéal pédagogique? Recherche en éducation, 20, 66-75.

Joule, R.-V. \& Beauvois, J.-L. (2009). La soumission librement consentie (6e éd.). Paris: Presses Universitaires de France.

Joule, R.-V \& Beauvois, J.-L. (2014). Petit traité de manipulation à l'usage des honnêtes gens (nouvelle éd. augmentée). Grenoble: Presses Universitaires de Grenoble.

Goody, J. (1979). La raison graphique. La domestication de la pensée sauvage. Paris: Minuit.

Henri-Panabière, G. (2008, novembre). Ascèse et planification: entre socialisation familiale et contraintes scolaires. In Symposium La constitution scolaire et extra-scolaire d'habitudes ou de dispositions scolairement évaluées (pp. 17-24). Université Rennes 2, colloque "Efficacité et équité en éducation» Communication non publiée, disponible à: https://esup.espe-bretagne.fr/efficacite_et_equite_en_education/programme/symposium_panabiere.pdf 
Hughes E. (1996). Le regard sociologique. Essais choisis. Textes rassemblés et présentés par Jean-Michel Chapoulie, Paris: Editions de l'EHESS.

Keddie, N. (1971/2007). Le savoir dispensé dans la salle de classe». In J. Deauvieau et J.P. Terrail (Éd.), Les sociologues, l'école et la transmission des savoirs (pp. 151-187). Paris: La Dispute.

Lahire B. (1998) L'homme pluriel. Les ressorts de l'action. Paris: Nathan

Lahire, B. (1995). Tableaux de famille. Paris: Gallimard Le Seuil.

Lahire, B. (2005). L'esprit sociologique. Paris: La découverte.

Mauger, G. (2001). Les politiques d'insertion. Une contribution paradoxale à la déstabilisation du marché du travail. Actes de la recherche en sciences sociales, 136-137, 5-14.

Millet, M. \& Thin, D. (2005). Le temps des familles populaires à l'épreuve de la précarité. Lien social et politique, 54, 153-162.

Monchatre, S. (2004). De l'ouvrier à l'opérateur: chronique d'une conversion. Revue française de sociologie, 45, 69-102.

Plaisance, E. (1986). L'enfant, la maternelle, la société. Paris: PUF.

Terrail, J.-P. (1984). Familles ouvrières, école, destin social (1880-1980). Revue française de sociologie, $25,3,421-436$.

Verret, M. (1975). Le temps des études. Paris: H. Champion.

Mots-clés: Rapport au temps, école primaire, autonomie, inégalités, socialisation

\section{Wenn der Schüler seine Zeit verwaltet: Die Zeit des selbständigen Arbeitens in der Grundschule}

\section{Zusammenfassung}

Der Beitrag diskutiert die zeitlichen Widersprüche, mit denen Schülerinnen und Schülern sowie Grundschullehrpersonen im Rahmen des Selbstständigen Lernens konfrontiert werden. Er untersucht die Formen des Bezugs zur Zeit, die durch diese Arbeitsmethoden untermauert werden. Auf der Grundlage einer ethnographischen Untersuchung, die in einer Grundschule in Genf durchgeführt wurde, geht klar hervor, dass Lernende einen Zeitplan erstellen müssen, der gleichzeitig Ausgangspunkt oder Mittel von Unabhängigkeit und Kontrolle ist. Der Artikel untersucht, wie zeitliche Kategorien verwendet werden, um die Verhaltens- und kognitiven Schwierigkeiten der Schüler zu naturalisieren, auch wenn man diese als Ergebnis von zeitlicher Sozialisation im familiären Umfeld interpretieren kann, die der Schule zugutekommen.

Schlagworte: Beziehung zur Zeit, Grundschule, Autonomie, Ungleichheit, Sozialisation 


\section{Quando I'alunno gestisce il suo tempo: quale rapporto al tempo durante il lavoro autonomo nella scuola elementare}

\section{Riassunto}

Questo contributo affronta le contraddizioni temporali che sono imposte agli alunni delle scuole elementari e agli insegnanti nei programmi di lavoro autonomo. Esso esamina le modalità di relazione con il tempo sottointese a questi metodi di lavoro. Basato su un'indagine etnografica condotta in una scuola elementare di Ginevra, esso evidenzia che gli studenti devono stabilire una relazione manageriale con il tempo, fonte sia di emancipazione che di controllo. Il nostro contributo analizza come le categorie temporali vengono utilizzate per naturalizzare le difficoltà comportamentali e cognitive degli studenti, anche se possono essere interpretate come il prodotto di socializzazioni temporali familiari ugualmente legittime e redditizienel nel contesto della scuola, in maniera inuguale.

Parole chiave: Relazione con il tempo, scuola elementare, autonomia, disuguaglianza, socializzazione

\section{When the student manages his time: the relation to time in autonomous work at primary school}

\section{Summary}

This contribution highlights the temporal contradictions among pupils and teachers in primary school in the context of autonomous work. It examines the relation to time underpinned by these working modalities. Based on an ethnographic survey carried out in a primary school in Geneva, it highlights that students have to establish a managerial relationship at the time, which is a source of both emancipation and control. It considers how temporal categories are used to naturalize students' behavioral and cognitive difficulties. It shows how pupils are unequal in terms of school temporality, depending on their family environment.

Keywords: Relation to time, primary school, autonomy, inequalities, socialization 